\title{
Somatosensory test responses in children with growing pains
}

\author{
This article was published in the following Dove Press journal: \\ Journal of Pain Research \\ I December 201 I \\ Number of times this article has been viewed
}

\author{
Shanthi Pathirana' \\ David Champion ${ }^{1,2}$ \\ Tiina Jaaniste \\ Anthony Yee ${ }^{2}$ \\ Cindy Chapman' \\ 'Department of Anesthesia and \\ Pain Medicine, ${ }^{2}$ Department of \\ Rheumatology, Sydney Children's \\ Hospital, Randwick, New South \\ Wales, Australia
}

Correspondence: David Champion Department of Anesthesia and Pain Medicine, Sydney Children's Hospital, High St, Randwick, NSW 203 I Australia Tel +6I 29382 I 585

Fax +6I 299044900

Email dchamp@bigpond.net.au
Purpose: To further the understanding of growing pains (GP), in particular, the nature of this pain disorder.

Methods: This study included 33 children aged 5-12 years who met criteria for GP (cases) and 29 children without GP of similar age and sex (controls). Nineteen controls were siblings of cases. GP was diagnosed by standard consensus questionnaires. A questionnaire addressed characteristics of the pain and family history of GP. Evidence for peripheral neuropathic disorder was tested by somatosensory testing and provocation tests of peripheral nerves. Somatosensory testing by a blinded researcher involved threshold determination and/or response magnitude to nonpainful stimuli including touch, dynamic brush, cold, vibration, and deep pressure applied to limb and abdominal sites.

Results: Distributional, temporal, and quality characteristics of the pain were in accordance with published descriptions. There was no indication of primary musculoskeletal disorder. No evidence was found that GP is a peripheral neuropathic pain syndrome. There were minor but statistically significantly increased responses to cutaneous cold, vibration, and to deep pressure stimuli in cases compared to controls, evident in a wider distribution than the symptomatic lower limbs.

Conclusion: GP is a regional pain syndrome with evidence in this study of mild widespread disorder of somatosensory processing.

Keywords: growing pains, somatosensory testing, case-control study

\section{Introduction}

From the time of the earliest descriptions of growing pains (GP) to the most recent reviews, ${ }^{1-3}$ the nature of the prevalent disorder of childhood, GP (benign nocturnal limb pains of childhood), has remained enigmatic. Early etiological theories of GP addressed growth, ${ }^{4}$ relative hyperactivity and fatigue, ${ }^{5-8}$ anatomical/biomechanical factors, ${ }^{9,10}$ and psychogenic factors. ${ }^{4,11}$ However, none of these factors have been shown to be more than potential contributors. The current study was designed to shed light on the question put forward by Naish and Apley, "What is this malady called GP?"4

Evidence that GP may be arising from somatic tissues, particularly musculoskeletal structures, in the limbs has not been convincing. ${ }^{1,3}$ The possibility that GP is mediated by peripheral nerve dysfunction has not been definitively tested. Evidence of disordered somatosensory processing in the form of widespread deep pressure allodynia (pain due to a stimulus which does not normally provoke pain)/hyperalgesia (an increased response to a stimulus which is normally painful) has been demonstrated by pain threshold testing by Hashkes et al. ${ }^{12}$ This is the only published study of somatosensory 
testing in GP and did not include responses to cutaneous stimuli which are less sensitively elicited in clinical conditions characterized by a widespread deep hyperalgesic state. The hypothesis of this paper is based on Haschkes et al's evidence for deep hyperalgesia, with the aim of confirming this hypothesis and extending the evidence to cutaneous somatosensory responses.

Arising from this background, the hypothesis that GP is characterized by widespread disorder of somatosensory processing was tested. Alternatively, it is a peripheral neuropathic pain syndrome (pain initiated or caused by a primary lesion or dysfunction in the (peripheral) nervous system) ${ }^{43}$

\section{Materials and methods}

Thirty-three children (14 males, 19 females) aged 5-12 years were recruited by advertisement (newspaper, radio, school newsletter, hospital notice board), conscious that the peak age of point prevalence for GP is 4-6 years. ${ }^{8}$ However, symptoms may develop later and continue into adolescence, ${ }^{35}$ and somatosensory test responses are less reliable under 5 years. ${ }^{23}$ The written advertisements were headlined: "Volunteers, children aged 5-12 years, are required for an ethically approved study into the nature of nocturnal limb pain syndrome (GP)." The content included: "This study will be conducted by personnel from the Sydney Children's Hospital (Pain Medicine Unit) and the University of New South Wales. If your child has aches and pains in the limbs, especially legs and particularly at night, participation in this study would be greatly appreciated. This study is in no way harmful and should not cause distress. We shall also be asking for a volunteer, such as a friend, of similar age and same sex as your child to come along and participate in what will be an interesting study."

Those who responded were mailed a package containing further information about the study including the planned sensory testing, consent forms, and a GP screening questionnaire, ${ }^{13}$ based on Petersen's GP criteria (Table 1). ${ }^{14}$ With there being no gold standard for the diagnosis of GP, the Petersen criteria have been widely applied in published studies to a point of reasonable consensus. ${ }^{15} \mathrm{~A}$ case selection flowchart is presented as Figure 1. Each participant (cases and controls) was also reviewed by a pediatrician (AY) to consider differential diagnoses of lower limb pain.

The control group comprised 29 children (12 males, 17 females) who did not have limb pain. In view of the difficulties in recruiting volunteers to undergo somatosensory testing, 19 controls were siblings of cases without GP, eight of whom were unaffected twins (three monozygotic; five dizygotic). The high number of twins was the result of advertisements that, for purposes external to the current study, indicated a particular interest in twins.

A set of clinical somatosensory test procedures was developed based on the standard neurological clinical examination and selected somatosensory tests applicable to children in an office practice to obtain evidence about peripheral neuropathic disorders and/or abnormal central sensory processing. These methods were derived from published protocols. ${ }^{16-20}$ The selection of multimodal-stimulus response measures has been further supported recently by Neziri et al, ${ }^{21}$ who showed that responses to different modalities represent different specific dimensions and should be assessed in combination. The current study however did not use any noxious stimuli (pain provocation in children with a normally functioning nervous system) on request from the South Eastern Sydney Area Health Service Ethics Committee, who approved the final protocol. Thus, there were no pain threshold determinations. The procedures were demonstrated by the researcher (SP), who was blinded as to case or control status. The process was organized by the participating pediatrician so that the blinding was strictly applied.

Taking into consideration the avoidance of quantitative sensory testing apparatus requiring a laboratory base, the related portability and time availability for office/bedside application, and the avoidance of pain thresholds for heat, cold, and mechanical stimuli, the following test stimuli (and responses) were selected and applied to specified sites. Additionally, the cases and controls were assessed for abnormal responses in peripheral nerve or dermatomal distribution. The procedures are summarized in Table 2.22-25

\section{Test stimuli}

With focus on the distribution of quantitative or qualitative abnormalities, static light touch was assessed by nonstroking touch of obliquely applied soft brush fibers to lower legs, forearms, and abdomen. This was the first assessment of A-beta peripheral sensory channel, central lemniscal pathway, and a large array of cortical regions.

Calibrated von Frey monofilament stimuli (VF1 Optihair-2 [set of 12 optical glass filaments with force range 0.25-512 mN]; Marstock, Marburg, Germany) enabled fixed graded stimuli to assess static light touch. This procedure also assessed A-beta peripheral sensory channels and lemniscal central pathway. Applied to lower legs, forearms, and abdomen, it provided mechanical detection threshold for touch, pain threshold in states of cutaneous hyperalgesia. For touch 
Table I Definition of Petersen's "growing pains,"/2 modified by Evans and Scutter ${ }^{13}$

\begin{tabular}{|c|c|c|}
\hline Pain factors & Inclusion criteria & Exclusion criteria \\
\hline \multirow[t]{2}{*}{ Nature of pain } & Intermittent & Persistent \\
\hline & Some pain free days and nights & Increasing intensity \\
\hline Unilateral or bilateral & Bilateral & Unilateral \\
\hline \multirow[t]{2}{*}{ Location of pain } & Anterior thigh, calf, & Joint pain \\
\hline & posterior knee - in muscles & \\
\hline Onset of pain & Late afternoon or evening & Pain still present next morning \\
\hline \multirow[t]{3}{*}{ Physical examination } & Normal & Swelling, erythema, tenderness \\
\hline & & Local trauma or infection. Reduced joint range or motion \\
\hline & & Limping \\
\hline Laboratory tests & Normal & Objective findings eg ESR, x-ray, bone scan abnormalities \\
\hline Limitation of activity & Nil & Reduced physical activity \\
\hline
\end{tabular}

threshold, the method of limits was used with the average of threshold estimations from two tests commencing with a subthreshold force $(0.25 \mathrm{mN})$ and two tests commencing with a suprathreshold force $(64 \mathrm{mN})$. Mean stimulus-response determinations from the same sites to von Frey filament number 9 were determined using the Color Analog Scale (0-10) with upper anchor "very strong touch."

Repetitive dynamic brush stimuli were applied to the limbs and abdomen (as control site) to determine the presence of cutaneous hyperesthesia or dynamic mechanical allodynia.

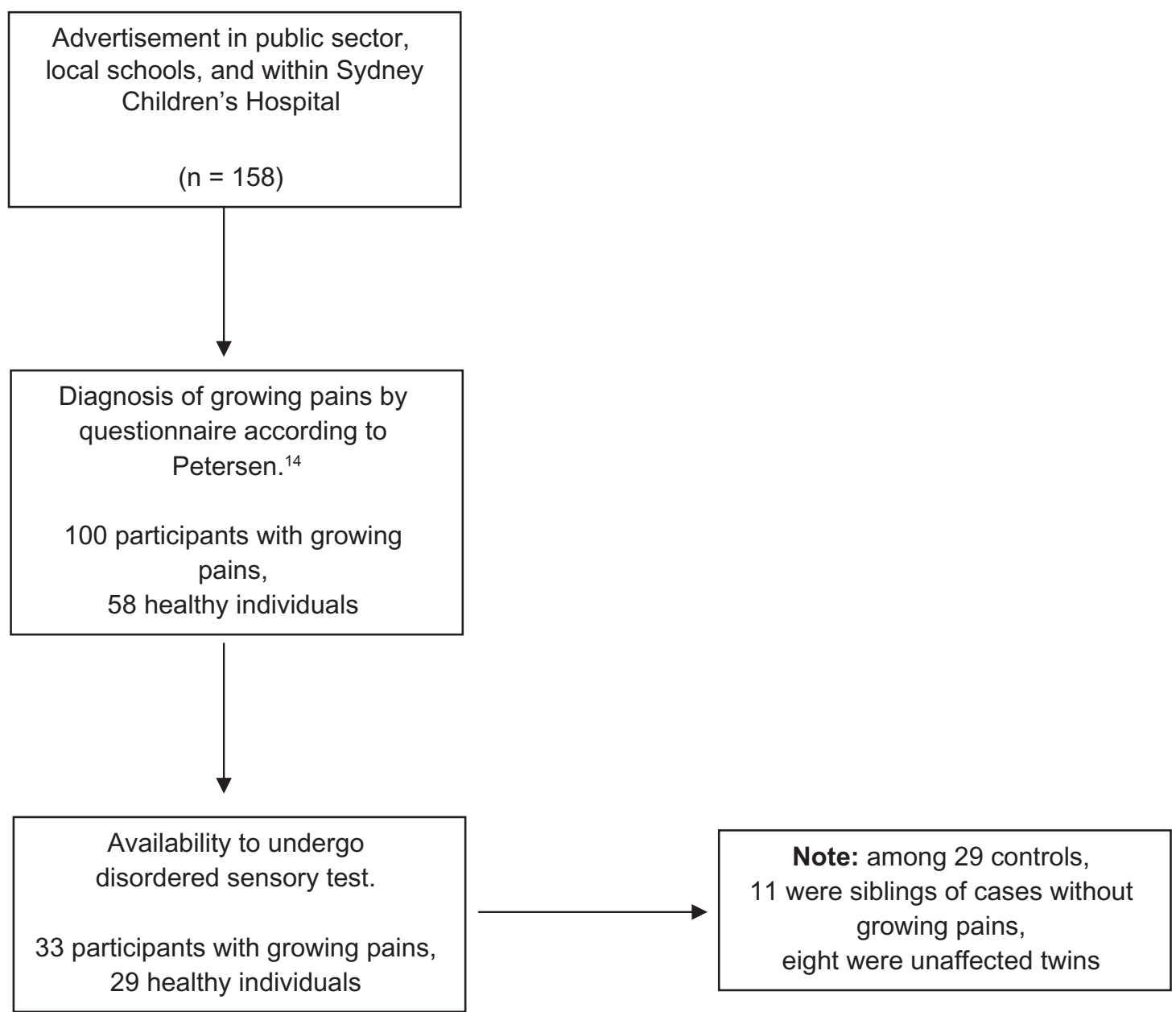

Figure I Case selection flowchart. 


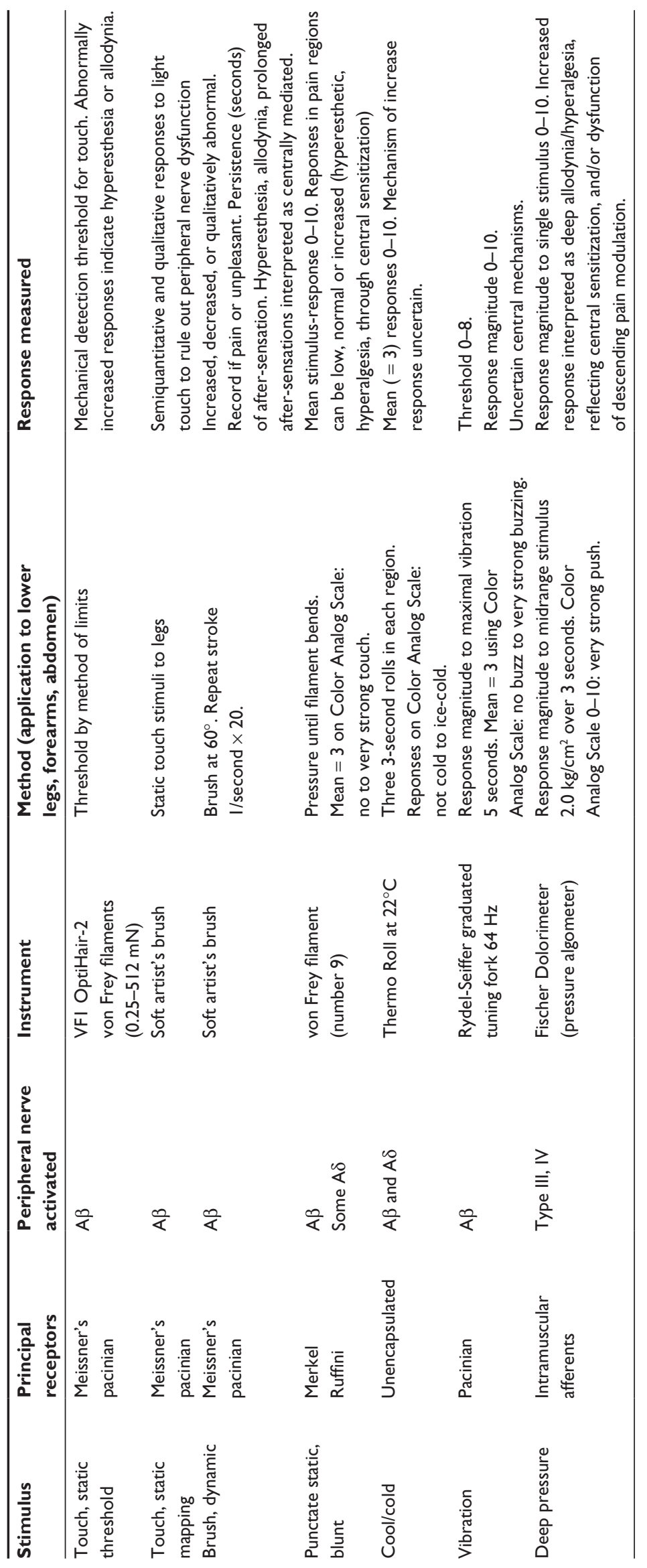


The brushing activates A-beta peripheral sensory channel and lemniscal sensory pathway. The dynamic repetitive stimuli test for temporal summation effects which correlate better with neuropathic pain states than single static stimuli. A standardized camel hair brush (SENSE Lab ${ }^{\mathrm{TM}}$ Brush-05; Somedic, Horby, Sweden) exerting a force of approximately $200-400 \mathrm{mN}$ was applied with a single stroke of approximately $2 \mathrm{~cm}$ in length over the skin in runs of ten per test site.

Rydel-Seiffer graduated tuning fork $(64 \mathrm{~Hz}, 8 / 8$ scale; US Neurologicals, Poulsbo, WA) was applied to bony prominences, tibial tuberosities, olecranons, and skin over soft tissues (lower legs, forearms, and abdomen). Vibration assessed the A-beta peripheral sensory channel and central lemniscal pathway. Vibration threshold was determined as a disappearance threshold (method of limits). A mean stimulusresponse determination for the maximal vibration intensity was determined on a $0-10$ scale using an adapted Color Analog Scale with upper anchor "very strong buzzing."

The assessment of somatosensory responses to cool stimuli was determined by a metal thermoRoll (Marstock). Cool/ cold stimuli activate A-beta and A-delta peripheral sensory channels and spinothalamic central pathways. Three rolls of 3 seconds were made at each limb and abdomen site. The roller was allowed to equilibrate with room temperature set at $22^{\circ} \mathrm{C}$. The upper anchor of the Color Analog Scale (0-10) was "ice cold."

Responses to blunt deep pressure stimuli were assessed by application of the Fischer pressure algometer (FDK 10; PDT Inc, NY) to consistent sites over deep somatic tissues (middle lower legs posterolaterally, middle forearms posterolaterally, abdomen, tibial tuberosities, and olecranons). This device has been used in children. ${ }^{12,26}$ Blunt deep (muscle) pressure, as it increases, activates intramuscular afferents Type III and IV and the spinothalamic central pathway. Responses to deep force reaching $2 \mathrm{~kg} / \mathrm{cm}^{2}$ over 3 seconds, a level comfortably below the mean pain threshold in healthy children, were obtained using the Color Analog Scale (0-10) with adapted anchors, the upper being "very strong push."
The Color Analog Scale $^{27}$ was applied with anchors modified in accordance with the type of stimulus. The Color Analog Scale has been shown to be reliable and valid in pain contexts in children as young as 5 years. ${ }^{28-30}$ Generally, visual analog scales have also been used in diverse nonpain applications.

Children were assessed for abnormal mechanosensitivity of peripheral nerves with a stretch by straight leg raising ${ }^{31}$ and brachial plexus provocation tests. ${ }^{32}$

Student's $t$-tests for independent samples were performed to compare cases and controls for all sensory testing measures. For each sensory modality, each tested site was analyzed as well as the mean across all tested sites.

\section{Results}

There were no significant demographic or anthropometric differences between cases and controls (Table 3 ). In the GP case sample $(\mathrm{n}=33)$, pain was present in the knee region and lower leg in all cases, additionally in the upper leg in eight cases $(24 \%)$, and in the arms in three cases (9\%). Pain was described as aching in 30 cases (94\%), deep in 13 (39\%), and there were accompanying pins and needles in nine (27\%). Episodes occurred daily in six cases (19\%), weekly in nine $(28 \%)$, monthly in eleven (34\%), and less frequently in six (19\%). The most common duration of a pain episode was 30-60 minutes. Pain intensity was minimal in the mornings and maximal at night. A total of 24 parents (72\%) of children with GP reported that their child experienced pain of sufficient severity to wake the child and 22 (66\%) reported that the pain induced crying.

There was no clinical evidence of a peripheral neuropathic pain syndrome with there being no indication of sensory impairment in peripheral nerve or dermatome distribution, no neurological motor deficits, and no abnormal mechanosensitivity of peripheral nerves according to sciatic and brachial plexus stretch maneuvers.

There were no significant differences in somatosensory test responses between cases and controls in the lower leg

Table 3 Clinical characteristics of participants

\begin{tabular}{|c|c|c|c|c|}
\hline & Cases $(\mathbf{N}=33)$ & Controls $(\mathbf{N}=\mathbf{2 9})$ & t-value & $P$ \\
\hline Age (years) mean $\pm S D$ & $7.9 \pm 2.3$ & $8.0 \pm 2.2$ & 0.05 & 0.96 \\
\hline Sex (male/female) & $14 / 19$ & $12 / 17$ & 0.08 & 0.94 \\
\hline Height $(\mathrm{cm})$ mean $\pm S D$ & $13 \mid \pm 15$ & $129 \pm 12$ & 0.65 & 0.52 \\
\hline Height percentile mean \pm SD & $75 \pm 25$ & $70 \pm 24$ & 0.69 & 0.49 \\
\hline Weight $(\mathrm{kg})$ mean $\pm \mathrm{SD}$ & $30 \pm 10$ & $28 \pm 8$ & 0.73 & 0.47 \\
\hline Weight percentile mean $\pm S D$ & $75 \pm 23$ & $73 \pm 19$ & 0.39 & 0.70 \\
\hline $\mathrm{BMI}\left(\mathrm{kg} / \mathrm{m}^{2}\right)$ mean $\pm \mathrm{SD}$ & $17 \pm 3$ & $17 \pm 2$ & 0.15 & 0.88 \\
\hline
\end{tabular}

Abbreviations: BMI, body mass index; SD, standard deviation. 
sites (except for pressure response magnitude at the lateral calf $[P=0.04])$, nor in the upper limbs or abdominal site.

Table 4 shows the somatosensory test results averaged across all sites. Response magnitudes to cold, vibration, and deep pressure stimulation were significantly greater in cases than controls while for static punctate tactile magnitude the apparent greater sensitivity in the cases did not reach statistical significance. There were no significant differences between cases and controls in touch threshold and vibration threshold. There was a nonsignificant trend to prolonged brush-induced after-sensations in cases.

\section{Discussion}

The study was designed to investigate the nature of the pain, specifically to determine whether there was regional (lower limb) or widespread disorder of somatosensory test responses, in children with GP. The diagnosis of GP is based on history, examination, and the exclusion of identifiable musculoskeletal/orthopedic, neurological, or other causes of limb (especially leg) pains. The features are characteristic of, and are reflected in, the criteria derived from Petersen. ${ }^{14}$ Criteria have been determined by gradually acquired consensus but with there being no gold standard for diagnosis, specificity has not been adequately validated. Because patients were accrued by advertisement, the extent to which their features would be similar to a random sample cannot be determined and thus extrapolation to the population of children with GP requires caution.

Consistent with previous studies and reviews, ${ }^{1-3}$ and consistent with the criterion-based, widely-applied GP definition, ${ }^{14}$ there was no evidence for a musculoskeletal/ somatic tissue disorder. Further, the essentially negative responses to the neuropathic pain questionnaire, the absence of sensory impairment in peripheral nerve or dermatomal distribution, and the lack of abnormal mechanosensitivity to brachial plexus and lumbosacral nerve root (sciatic) provocation provided no support for the interpretation of a peripheral neuropathic pain syndrome. ${ }^{33,34}$

The investigator-blind somatosensory testing covered a range of stimulus modalities. Although the lower leg cutaneous testing showed consistent trends to more sensitive responses in cases than controls, these differences did not reach statistical significance. However, the greater response to deep pressure in the soft tissues of the lower legs in children with GP compared with responses in control children was statistically significant, consistent with previous evidence of deep pressure allodynia/hyperalgesia in children with GP. ${ }^{12,35}$ The overall greater sensitivity of responses averaged across all sites (statistically significant for response magnitude to cutaneous cold, vibration, and deep pressure) suggests a mild widespread disorder of somatosensory processing.

The reference values for children in the relevant age range for face, hand, and foot sites, applying the German Research Network on Neuropathic Pain protocol, ${ }^{20}$ were reported in detail by Blankenburg et al. ${ }^{23}$ The present somatosensory test protocol was in accord with procedures in the Blankenburg et al study ${ }^{23}$ and in studies which the authors cited, however, it was simpler, avoided pain threshold, and added response magnitude tests. These investigators found that the younger children (6-8 years) were generally less sensitive to thermal and mechanical detection stimuli but more sensitive to pain stimuli than older children (9-12 years), who in turn were similar to adolescents (13-17 years). The sample size was insufficient to permit valid subset analysis. The response magnitude estimations to subpain stimuli using the Color Analog Scale were novel. These punctuate pressure (blunt, static), vibration intensity, and deep (blunt, static) stimulus procedures were used in general by those investigators, but not in the stimulus response manner the present study applied to avoid pain threshold testing.

Table 4 Somatosensory test responses (all sites combined)

\begin{tabular}{|c|c|c|c|c|c|c|}
\hline \multirow[t]{2}{*}{ Sensory stimulus test } & \multicolumn{2}{|l|}{ Mean (SD) } & \multirow[t]{2}{*}{$t(60)$} & \multirow[t]{2}{*}{$\mathbf{P}$} & \multirow[t]{2}{*}{$95 \% \mathrm{Cl}$} & \multirow[t]{2}{*}{ ES } \\
\hline & $\begin{array}{l}\text { Cases } \\
(N=33)\end{array}$ & $\begin{array}{l}\text { Controls } \\
(N=29)\end{array}$ & & & & \\
\hline Touch threshold $(\mathrm{mN})$ & $8.02(13.96)$ & $8.86(17.50)$ & -0.21 & 0.83 & $(-8.84,7.16)$ & 0.03 \\
\hline Vibration threshold (0-8) & $7.45(0.56)$ & $7.5 I(0.4 I)$ & -0.46 & 0.65 & $(-0.31,0.19)$ & 0.06 \\
\hline Brush after-sensations (seconds) & $2.51(3.85)$ & $\mathrm{I} .4 \mathrm{I}(2.65)$ & 1.29 & 0.20 & $(-0.6 \mathrm{I}, 2.80)$ & 0.16 \\
\hline Punctate tactile magnitude $(0-10)$ & $4.74(1.75)$ & $4.18(1.74)$ & 1.25 & 0.22 & $(-0.33,1.45)$ & 0.16 \\
\hline Cold response magnitude $(0-10)$ & $6.37(1.30)$ & $5.52(1.86)$ & 2.07 & $0.04 *$ & $(0.03,1.69)$ & 0.26 \\
\hline Vibration response magnitude $(0-10)$ & $4.90(1.21)$ & $4.17(1.47)$ & 2.15 & $0.04 *$ & $(0.05,1.4 I)$ & 0.27 \\
\hline Pressure response magnitude $(0-10)$ & $5.96(1.06)$ & $5.12(1.76)$ & 2.25 & $0.03^{*}$ & $(0.09,1.60)$ & 0.28 \\
\hline
\end{tabular}

Note: *Significant at $P<0.05$.

Abbreviations: $\mathrm{Cl}$, confidence interval; $\mathrm{ES}$, effect size; $\mathrm{SD}$, standard deviation. 
Based on published evidence and results from this present study, GP does not appear to be a somatic/nociceptive (ie, musculoskeletal pain) syndrome, nor is it a peripheral neuropathic pain syndrome. The widespread distribution of sensory disturbances within this sample suggests the likelihood that underlying spontaneous pain is central sensitization of nociception $^{36}$ and/or disordered descending pain modulation in the central nervous system. ${ }^{37}$ The authors suggest that, on the basis of this and the authors' previous studies, and other published studies, GP can be provisionally allocated to the group of idiopathic pain disorders as defined by Diatchenko et $\mathrm{al}^{38}$ and to the functional pain syndromes as defined by Mayer and Bushnell. ${ }^{39}$ These disorders are characterized by an absence of definable somatic disease, by comorbid associations with other idiopathic pain syndromes, by indicators of disordered central processing of nociception, genetic influence, often neuroendocrine dysfunction, and by psychosocial associations. As a group they are prevalent, eg, 1-year prevalence of any syndrome in 5-7 year old children of $23.2 \%{ }^{40}$

There are a number of limitations in this study. Given the modest sample size, the multiple statistical comparisons should be acknowledged as a point of caution regarding extrapolation. The diagnosis of GP was based on consensus criteria and the exclusion of other conditions. This is a limitation in all studies on GP at present. An important objective of future studies is to work towards improved phenotype definition. The sample was pragmatically obtained rather than derived from a random survey. There was potential for selection bias based on severity and greater parental awareness. The controls included siblings, of whom a disproportionate number were twins; however, the reasonable expectation is that such selection would tend to reduce contrast in somatosensory responses in cases and controls.

The somatosensory test methods used in this study are relatively novel and have only recently been applied to children. The somatosensory test procedures were conservative in that they involved the quantification of responses to subpain threshold stimuli whereas quantitative sensory testing has been used most extensively in the assessment of pain thresholds or the quantification of responses to suprathreshold stimuli. ${ }^{41}$ Suprathreshold measures (tolerance) are believed to be more clinically relevant than threshold measures. ${ }^{42}$ Temporal summation, a dynamic psychophysical measure, is thought to better capture the pain modulatory ability of the central nervous system as compared to state measures, such as threshold and tolerance that only measure a single point in the pain processing continuum..$^{43}$ Thus, for most of the measures, the contrast in responses between cases and controls were relatively minor although the direction consistently favored greater sensitivity of cases for all modalities. A greater contrast between cases and controls would be expected from deep repetitive pressure stimuli testing for temporal summation, radiation, and persistent after-sensations.

\section{Conclusion}

The current study has shown that the prevalent pain syndrome of childhood, GP, is characterized by widespread mildly disordered (relatively sensitive) cutaneous and deep somatosensory responses to several stimulus modalities. The perspective of the authors is that although GP has been considered an "end-organ" pain syndrome, an idiopathic/ functional pain syndrome concept is favored..$^{39}$

\section{Disclosure}

This research was supported by Sydney Children's Hospital Foundation, Faculty of Medicine, University of New South Wales.

\section{References}

1. Evans AM. Growing pains: contemporary knowledge and recommended practice. J Foot Ankle Res. 2008;1(1):4.

2. Lowe RM, Hashkes PJ. Growing pains: a noninflammatory pain syndrome of early childhood. Nat Clin Pract Rheumatol. 2008;4(10):542-549.

3. Foster HE, Boyd D, Jandial S. "Growing pains." A practical guide for primary care. Reports on the Rheumatic Diseases Series 6. 2008;(1):1-6.

4. Naish JM, Apley J. "Growing pains": a clinical study of non-arthritic pains in children. Arch Dis Child. 1951;26(126):134-140.

5. Brenning R. Growing pains. Acta Soc Med Ups. 1960;65:185-201.

6. Baxter MP, Dulberg C. Growing pains in childhood: a proposal for treatment. J Pediatr Orthop. 1988;8(4):402-406.

7. Friedland O, Hashkes P, Jaber L, et al. Decreased bone strength in children with growing pains as measured by quantitative ultrasound. J Rheumatol. 2005;32(7):1354-1357.

8. Evans AM, Scutter SD, Lang LMG, Dansie BR. "Growing pains" in young children: a study of the profile, experiences and quality of life issues of four to six year old children with recurrent leg pain. The Foot. 2006;16(3):120-124.

9. Evans AM. Relationship between "growing pains" and foot posture in children: single-case experimental designs in clinical practice. $J \mathrm{Am}$ Podiatr Med Assoc. 2003;93(2):111-117.

10. Hawksley J. The nature of growing pains and their relation to rheumatism in children and adolescents. Br Med J. 1939;1(4073):155-157.

11. Oberklaid F, Amos D, Liu C, Jarman F, Sanson A, Prior M. "Growing pains": clinical and behavioral correlates in a community sample. J Dev Behav Pediatr. 1997;18(2):102-106.

12. Hashkes P, Friedland O, Jaber L, Cohen H, Wolak B, Uziel Y. Children with growing pains have decreased pain threshold. J Rheumatol. 2004;16(3):610-613.

13. Evans AM, Scutter SD. Development of a questionnaire for parental rating of leg pain in young children: internal validity and reliability testing following triangulation. The Foot. 2004;14(1):42-48.

14. Petersen H. Growing pains. Pediatr Clin North Am. 1986;33(6): 1356-1372.

15. Asadi-Pooya AA, Bordbar MR. Are laboratory tests necessary in making the diagnosis of limb pains typical for growing pains in childen? Pediatr Int. 2007;49(6):833-835. 
16. Chong PS, Cros DP. Technology literature review: quantitative sensory testing. Muscle Nerve. 2004;29(5):734-747.

17. Cruccu G, Anand P, Attal N, et al. EFNS guidelines on neuropathic pain assessment. Eur J Neurol. 2004;11(3):153-162.

18. Harden RN. Chronic neuropathic pain. Mechanisms, diagnosis, and treatment. Neurolgist. 2005;11(2):111-122.

19. Rolke R, Magerl W, Campbell KA, et al. Quantitative sensory testing: a comprehensive protocol for clinical trials. Eur J Pain. 2006;10(1):77-88.

20. Rolke R, Baron R, Maier C, et al. Quantitative sensory testing in the German Research Network on Neuropathic Pain (DFNS): standardized protocol and reference values. Pain. 2006;123(3):231-243.

21. Neziri AY, Curatolo M, Nuesch E, et al. Factor analysis of responses to thermal, electrical, and mechanical painful stimuli supports the importance of multi-modal pain assessment. Pain. 2011;152(5):1146-1155.

22. Backonja MM, Walk D, Edwards RR, et al. Quantitative sensory testing in measurement of neuropathic pain phenomena and other sensory abnormalities. Clin J Pain. 2009;25(7):641-647.

23. Blankenburg M, Boekens $H$, Hechler $\mathrm{T}$, et al. Reference values for quantitative sensory testing in children and adolescents: developmental and gender differences of somatosensory perception. Pain. 2010;149(1):76-88.

24. Haanpaa ML, Backonja MM, Bennett MI, et al. Assessment of neuropathic pain in primary care. Am J Med. 2009;122(10 Suppl): S13-S21.

25. Walk D, Sehgal N, Moeller-Bertram T, et al. Quantitative sensory testing and mapping a review of nonautomated quantitative methods for examination of the patient with neuropathic pain. Clin J Pain. 2009;25(7):632-640.

26. Metsahonkala L, Anttila P, Laimi K, et al. Extracephalic tenderness and pressure pain threshold in children with headache. Eur J Pain. 2006;10(7):581-585.

27. McGrath P, Seifert CE, Speechley KN, Booth JC, Stitt L, Gibson MC. A new analogue scale for assessing children's pain: an initial validation study. Pain. 1996;64(3):435-443.

28. Bailey B, Bergeron S, Gravel J, Daoust R. Comparison of four pain scales in children with acute abdominal pain in a pediatric emergency department. Ann Emerg Med. 2007;50(4):379-383, 383. e1-2.

29. McConahay T, Bryson M, Bulloch B. Clinically significant changes in acute pain in a pediatric ED using the Color Analog Scale. Am J Emerg Med. 2007;25(7):739-742.
30. Bulloch B, Garcia-Filion P, Notricia D, Bryson M, McConahay T. Reliability of the color analog scale: repeatability of scores in traumatic and nontraumatic injuries. Acad Emerg Med. 2009;16(5):465-469.

31. Ridehalgh C, Greening J, Petty NJ. Effect of straight leg raise examination and treatment on vibration thresholds in the lower limb: a pilot study in asymptomatic subjects. Man Ther. 2005;10(2):136-143.

32. Selvaratnam PJ, Matyas TA, Glasgow EF. Noninvasive discrimination of brachial plexus involvement in upper limb pain. Spine (Phila Pa 1976). 1994;19(1):26-33.

33. Treede RD, Jensen TS, Campbell JN, et al. Neuropathic pain: redefinition and a grading system for clinical and research purposes. Neurology. 2008;70(18):1630-1635.

34. Geber C, Baumgärtner U, Schwab R, et al. Revised definition of neuropathic pain and its grading system: an open case series illustrating its use in clinical practice. Am J Med. 2009;122(10 Suppl):S3-S12.

35. Uziel Y, Chapnick G, Jaber L, Nemet D, Hashkes PJ. Five-year outcome of children with "growing pains": correlations with pain threshold. J Pediatr. 2010;156(5):838-840.

36. Latremoliere A, Woolf CJ. Central sensitization: a generator of pain hypersensitivity by central neural plasticity. J Pain. 2009;10(9): 895-926.

37. Bingel U, Tracey I. Imaging and CNS modulation of pain in humans. Physiology (Bethseda). 2008;23:371-380.

38. Diatchenko L, Nackley AG, Slade GD, Fillingim RB, Maixner W. Idiopathic pain disorders - pathways of vulnerability. Pain. 2006;123(3):226-230.

39. Mayer E, Bushnell M. Functional pain disorders: time for a paradigm shift? In: Mayer EA, Bushnell MC, editors. Functional Pain Syndromes: Presentation and Pathophysiology. Seattle: IASP Press; 2009:531-565.

40. Rask CU, Olsen EM, Elberling H, et al. Functional somatic symptoms and associated impairment in 5-7-year-old children: the Copenhagen Child Cohort 2000. Eur J Epidemiol. 2009;24(10):625-634.

41. Hansson P, Backonja M, Bouhassira D. Usefulness and limitations of quantitative sensory testing: clinical and research application in neuropathic pain states. Pain. 2007;129(3):256-259.

42. Edwards RR, Sarlani E, Wesselmann U, Fillingim RB. Quantitative assessment of experimental pain perception: multiple domains of clinical relevance. Pain. 2005;114(3):315-319.

43. Arendt-Nielsen L, Yarnitsky D. Experimental and clinical applications of quantitative sensory testing applied to skin, muscles and viscera. J Pain. 2009;10(6):556-572.
Journal of Pain Research

\section{Publish your work in this journal}

The Journal of Pain Research is an international, peer-reviewed, open access, online journal that welcomes laboratory and clinical findings in the fields of pain research and the prevention and management of pain. Original research, reviews, symposium reports, hypothesis formation and commentaries are all considered for publication.

\section{Dovepress}

The manuscript management system is completely online and includes a very quick and fair peer-review system, which is all easy to use. Visit http://www.dovepress.com/testimonials.php to read real quotes from published authors. 